\title{
Performance Measurements of Feature TraCKING AND HISTOGRAM BASED TRAFFIC CONGESTION AlgORITHMS
}

\author{
Ozgur Altun ${ }^{1}$ and Kenan Aksoy ${ }^{2}$ \\ Proline Bilisim Sistemleri, Istanbul, Turkey \\ ${ }^{1}$ Research and Development Engineer, \\ ${ }^{2}$ Research and Development Engineer,
}

\begin{abstract}
In this paper, feature tracking based and histogram based traffic congestion detection systems are developed. Developed all system are designed to run as real time application. In this work, ORB (Oriented FAST and Rotated BRIEF) feature extraction method have been used to develop feature tracking based traffic congestion solution. ORB is a rotation invariant, fast and resistant to noise method and contains the power of FAST and BRIEF feature extraction methods. Also, two different approaches, which are standard deviation and weighed average, have been applied to find out the congestion information by using histogram of the image to develop histogram based traffic congestion solution. Both systems have been tested on different weather conditions such as cloudy, sunny and rainy to provide various illumination at both daytime and night. For all developed systems performance results are examined to show the advantages and drawbacks of these systems.
\end{abstract}

\section{KEYWORDS}

Traffic Congestion, Feature Extraction, ORB, Histogram.

\section{INTRODUCTION}

Intelligent Transportation Systems (ITS) applies advanced technologies of computers and electronics to collect the parameters of vehicle traffic. These parameters provide effective and efficient management and safety traffic solutions while developing technologies about Traffic Surveillance Systems/Technologies (TSS/T). Traffic surveillance systems include the technologies in order to manage the vehicle traffic on the road.

The migration from rural areas has caused the increasing the population in the urban areas in the last half a century. However, the number of vehicle use has increased day by day. This increment has led to important problems such as congestion in the vehicle traffic while traveling from a place to another. Nowadays, the traffic congestion is one of today's most important problems on the whole world. The problems causing from traffic congestion bring about both environmental, social, mental and even economic issues. For this reason, many scientific expertise area such as psychology, sociology, many engineering branches deal with how to solve the problems and its affects causing from traffic congestion. Especially in the urban regions, people waste more time in the traffic. In order to decrease this wasted time, many traffic management techniques as a part of Traffic Surveillance System have been developed by researchers and road management authorities to prevent traffic congestion problem. 
The traffic congestion is a matter that can be expressed with economic development, moral values, humanitarian attitude and behaviors and generally solved by using technology. By using intelligent traffic management and technological advances intensively, a new generation system which involves intelligent roads and vehicles can be designed. The data obtained from intelligent traffic management system which includes roads and vehicles enable the analysis of traffic problems. This analysis results also provides us an information in order to regulate the traffic flow. However, decreasing on the traffic congestion problems provide advantages to solve its effects. Improving the quality of transportation, reduction in fuel consumption and also environmental damage play an active role in the regulation of human psychology, physiology and biology.

In this paper, our aim is to design a real time system for the purpose of detecting the traffic congestion state on the road, highways and critical junctions etc. In order to realize our aim, we have developed feature tracking based and histogram based Traffic Congestion Detection System (TCDS). Both of these methods will be implemented as a part of Traffic Surveillance System in order to process the camera streams in a real time manner. Therefore, we have made a comparison between these methods to determine the availability under a real time system. Execution performances of developed system have been pointed out. Also, advantages and drawbacks of these methods have been presented in this paper.

Many researchers are developing solutions on traffic congestion. Vipin Jain et. al develop an algorithm to detect congestion level in the road traffic [1]. In this work, Jain et. al design an algorithm based on the histogram of considered road. They used the histogram value to detect the congestion within the specified range. K.D. Peiris et. al. have studied to extract traffic parameters such as density, speed and type of vehicles[2]. In this work, they have used the count of the cars to provide traffic density on the road. Kostia Robert used to feature extraction to detect and track vehicles on the road [3].. In this work, traffic density information are obtained according to tracking activities. Benjamin Coifmana et. al. are used the feature extraction of vehicles to detect the density of the traffic [4].

This paper is organized as follows. In methodology section, we present the details of the developed algorithms. All algorithms and approaches are discussed step by step in details. In experimental results section, we present the performance results of these algorithms and conclude the paper.

\section{METHODOLOGY}

In this work, a real time system has been designed for the purpose of measuring and detecting traffic congestion by using camera streams which are placed on roads, junction and critical vehicle passages. This system will be integrated with wide range cameras which are connected to our Traffic Surveillance System. In order to create a robust traffic congestion detection system, some kind of region limitations have to be carried out in the wide range camera's field of view (FOV). As is known, wide range cameras can involve more irrelevant objects or parameters in its FOV and traffic congestion detection systems deal with only the actions and which are occurred on the road. Also, processing of the irrelevant parameters produces an undesired extra computational load. So that, the irrelevant parameters have to be eliminated from the FOV of the cameras. In order to perform this kind of elimination, a specific area, which is known in the literature as Region of Interest (ROI), is determined on the FOV before the system is executed. Both feature tracking and histogram based algorithms take into account the determined ROI to perform their own process. 


\subsection{Feature Tracking Based Traffic Congestion Detection}

Feature based traffic congestion detection algorithm have been developed based on ORB feature detection method [5]. As we mentioned before, developed algorithms are designed to run in real time. As is known, many feature detection methods have been presented in the literature such as ORB, SURF, SIFT, BRIEF etc. In this work, ORB method are used for the detection of the features on the image. ORB feature detection method contain within itself the combination of FAST (Features from Accelerated Segment Test) keypoint [6] detector and BRIEF (Binary Robust Independent Element Feature) descriptor [7] and for this reason it is called as ORB (Oriented FAST and Rotated BRIEF). Both of FAST and BRIEF are attractive because of their good performance and low cost [5]. In the developing process, we have applied SURF, BRIEF and ORB feature detection method to our algorithm to find out the execution times of these methods. According to these result, we have determined that ORB feature detection method is suitable for a real time application.

The block diagram of feature tracking based algorithm can be seen in Figure 1. The processes, which are seen as Offline and Online, are employed respectively.

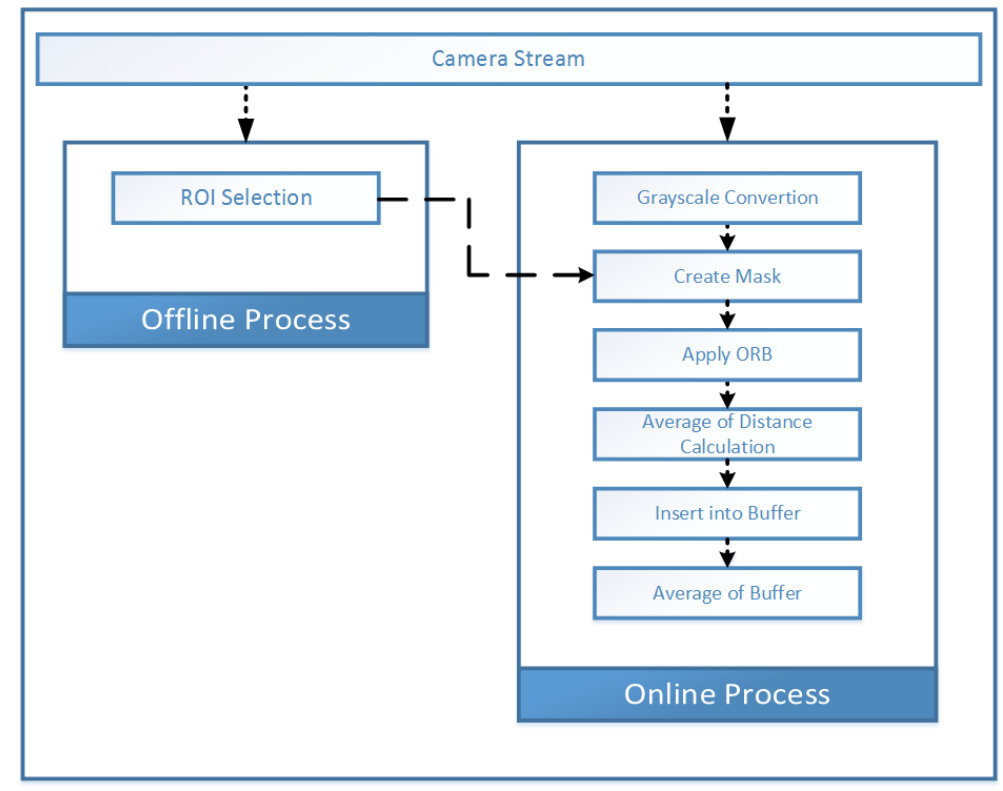

Figure 1 - Feature Tracking Based Traffic Congestion Algorithm

1) Offline Process: The offline process of this algorithm includes the determination of the road area as ROI. In order to perform this, the first frame which is presented from connected widerange camera are taken into account.

2) Online Process: In the online process, the frames which are consecutively obtained from connected camera's stream have been processed as depicted in Figure 1.

- Grayscale conversion: In order to avoid extra computation load arising from three band structure of colored frame, the grayscale conversion operation has been performed. This operation provide us fast processing for the next steps.

- Creating mask: The mask helps us to limit the area while applying the ORB feature method. As we mentioned before, this limitation produces us faster processing more than processing full image during the detection the of feature operation. This mask is generated by using determined ROI in the offline process. 
- Apply ORB: ORB feature detection operation includes three base steps which are detection keypoints, computing description and matching descriptions. This step is described in detail below.

- Detection Keypoints: Keypoints of an image are the reasonable corners on the image [5]. While applying the ORB feature detection method, FAST keypoint detection are used [6]. FAST takes one parameter, the intensity threshold between the center pixel and those in a circular ring about the center [5]. FAST keypoint detection method is described in detail in [6]. The ORB feature detection method uses FAST-9(circular radius of 9). However, in the ORB feature method, a Harris corner measure operation is employed onto detected keypoints. Harris corner measure operation put in order the keypoints. While implementing ORB feature, selected $\mathrm{N}$ of keypoint are used in order to provide the efficiency and increase the performance. In our application, the number of used keypoint has been defined as 500 .

- Computing Description: The ORB feature methods are used BRIEF descriptor in order to compute the description of detected keypoints. The description is used to speed up matching and reduce memory consumption. The BRIEF descriptor is a bit string description of an image patch constructed from a set of binary intensity test [5]. BRIEF description method is described in detail in [7].

- Matching Descriptions: Matching operation is used to find out the similarity of last two frame. For each frame, obtained features are matched respectively. This operation provide us tracking detected features between two obtained frames. After applying this operation, the information about matched features between previous and current frame are listed. By using this features, the migration distance can be calculated according to vehicle moving direction. The migration distance information is also related with the velocity of vehicles. If the velocity is high, the migration distance will be high or vice versa. In our algorithm, we have extracted traffic congestion information by using this information.

- Average of distance calculation: In this step, the average of all migrated point distances has been calculated. By performing this step, a single value is obtained to express the many migration distance of feature values.

- Insert into buffer: Buffer is used to keep obtained distance value. In our experiment, the size of this buffer have been defined as 30 .

- Average of buffer: The average of this buffer have been calculated to avoid instantaneous changing according to obtained instant values and cover erroneous average values. In our experiment, we have seen that unwanted features could affect the average migration distance calculation. In order to avoid this unwanted values and produce more accurate values, a buffer structure are used. However, this buffer denotes the average velocity in the traffic for a 30 frame. This values is equal to 1 second for our camera system.

\subsection{Histogram Based Traffic Congestion Detection}

Histogram is a method to find out the grayscale values distribution on the frame of an image. The grayscale value distribution changes for per frame while the vehicles are moving on the road. Generally, the histogram of the observed road are formed in a small portion of the values between 0 and 255. This values can vary according to environmental conditions. For examples, Vipin Jain et. al. [1] have experienced the histogram values of the roads in a day time conditions between 135 and 165 . According to this work, the congestion values on the road is changed based on this values. In our work, we have determined these values adaptively. In our work, two different methods are presented in order to determine traffic congestion state by using the calculated histogram of a frame. 


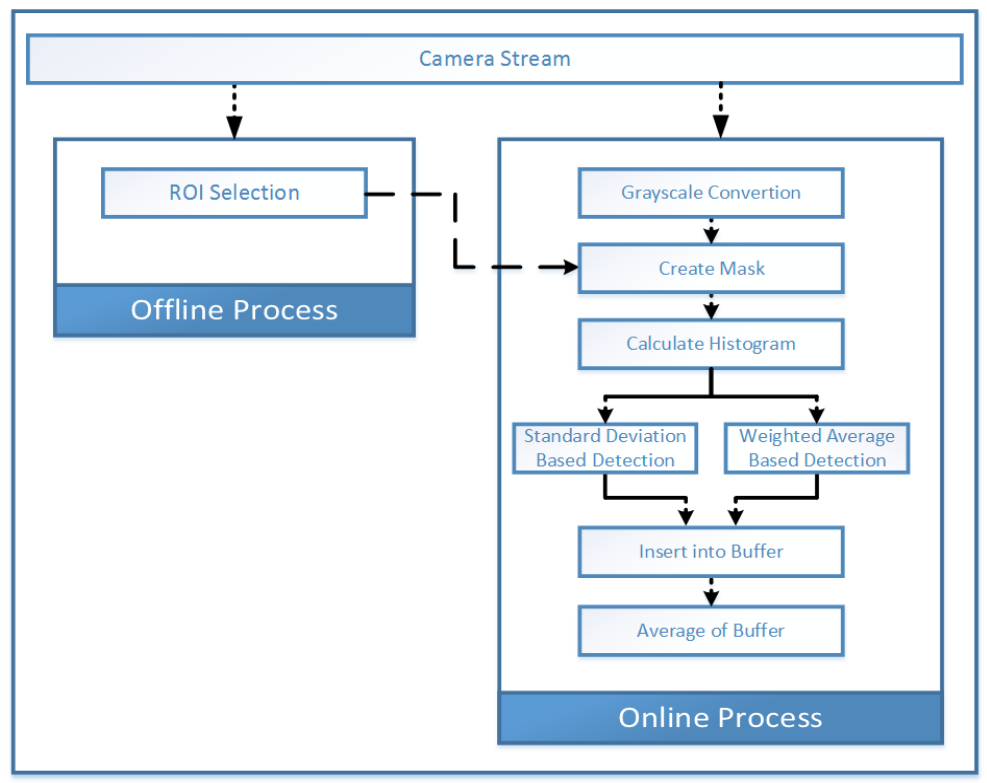

Figure 2. Histogram Based Traffic Congestion Algorithm

The block diagram of histogram based algorithm can be seen in Figure 2. This algorithm also includes both offline and online process as in feature based solution. However, ROI selection, grayscale conversion and create mask steps are applied in exactly the same manner as in the feature based solution. The other processes are expressed below.

- Histogram Calculation: As known, histogram is the distribution of the grayscale values of an image. In other words, histogram can be expressed as the count of the pixels at each different grayscale intensity value. In our project, due to the processing 8-bit grayscale image, the histogram of an image can be expressed with 256 different intensity level.

- Standard Deviation Based Detection: In this detection method, standard deviation and average value of the histogram are calculated. The ratio between the standard deviation and average values give us the instant traffic congestion level for an obtained image. For this purpose we have used the equation as defined in Equation.(1)

$$
I T R=\frac{\sigma}{\mu}
$$

\section{Equation 1 - Instant Traffic Ration}

where ITR is the Instant Traffic Ratio, $\sigma$ and $\mu$ are the standard deviation and average values respectively. $\sigma$ and $\mu$ values can be calculated with Equation (2) and Equation(3).

$$
\mu=\frac{1}{N} \sum_{i=0}^{N-1} p(i)
$$

Equation 2 - Average Values Calculation

$$
\sigma=\sqrt{\frac{1}{N} \sum_{i=0}^{N-1}(p(i)-\mu)^{2}}
$$

Equation 3 - Standard Deviation Calculation 
where $p(i)$ represents the histogram values of an image, $N$ is the number of element in the histogram In our work, $N$ is equal to 256.

Instant traffic ratio formula which is depicted in Equation (1), the standard deviation play main role on this calculation. The movement of the vehicles on the image affect the intensity of the grayscale level for each frame. If the vehicles are moving fast, the standard deviation and the histogram values changes at a high rate. On the other hand, the average value of histogram is used to calibrating the instant traffic level ratio. In our work, the processing of histogram is applied in the defined mask region. The region of the mask can be changes according to user selection. So that, the value of the standard deviation could not give the exact ratio to represent the instant traffic level. According to mask region selection by the user, the standard deviation value can get a value within the uncertain and wide range. In order to find out a general, narrow and certain range values for each mask size, the average value of the histogram are used. After obtaining the general values within the narrow range, we calibrate the values to detect traffic congestion level on the observed road.

- Weighted Average Based Detection: In this detection method, balance point which means center of gravity and average value of the histogram are calculated. This method are designed to take into account the values within \pm threshold range around the detected balance point. The ratio between summation of the values within the detected area and average of the histogram values give us the instant traffic congestion level for an obtained image. In order to find out the instant traffic ratio, we have used the equation as defined in Equation (4).

$$
\text { ITR }=\frac{X_{\text {Balance }}}{\mu}
$$

Equation 4 - Instant Traffic Ration

where $X_{\text {Balance }}$ represents the balance point of the calculated histogram. $X_{\text {Balance }}$ can be calculated with Equation (5).

$$
X_{\text {Balance }}=\frac{\sum_{n=0}^{N} Y(n) X(n)}{\sum_{n=0}^{N} Y(n)}
$$

Equation 5 - Balance Point Calculation

where $N$ is the number of element in the histogram, $Y(n)$ is the vertical values of the histogram, $X(n)$ is the horizontal values of the histogram.

\section{EXPERIMENTAL RESULTS}

In this paper we have developed algorithms to measure and detect traffic congestion. In this context, we have performed feature tracking based and histogram based TCDS. Also, we have presented the performance of these solutions. In order to measure the performance of the developed solutions different kinds of environmental conditions have been considered. Moreover, CPU cycle and CPU time performance have been measured. All CPU performance tests have been performed on the test environment as shown in Table I. Additionally, in the development process, $\mathrm{C}++$ and OpenCV library are used. In order to obtain camera streams, mobile electronic tracking system which name is MOBESE in TURKEY. In order to test the system all camera streams are provided by Proline Bilisim Sistemleri infrastructure. 
International Journal of Computer Science \& Information Technology (IJCSIT) Vol 7, No 2, April 2015

\begin{tabular}{|c|c|}
\hline CPU & Intel Xeon E5-2630 \\
\hline CPU Frequency & $2.6 \mathrm{GHz}$ \\
\hline CPU Core Count & 2 \\
\hline Instruction Set & $64-$ bit \\
\hline RAM & $16 \mathrm{~GB}$ \\
\hline Operating System & 64-bit Windows 7 Professional \\
\hline
\end{tabular}

Table 1 - TEST Environment

To show the real-time performance of developed algorithms, CPU clock cycle and execution time of per frame have been measured. Feature tracking and histogram based solutions CPU clock cycle and execution time performances are depicted on Figure 3(a,b) and Figure 4(a,b).

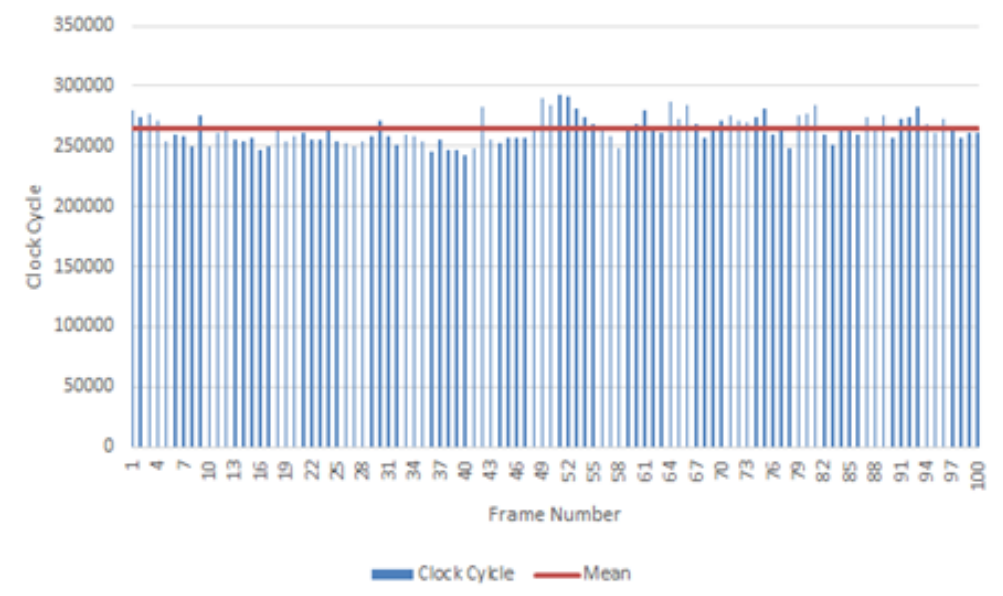

Figure 3.a - CPU Clock Cycle Performance of Feature Tracking Based TCDS

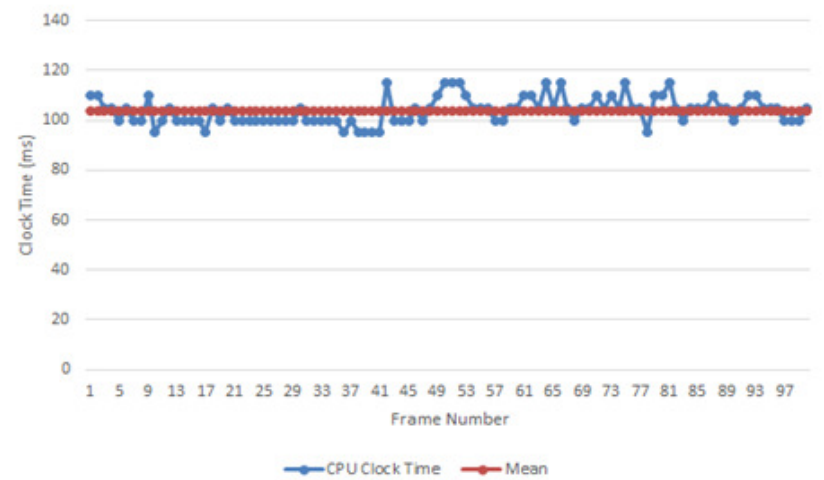

Figure 3.b - Execution Time Performance of Feature Tracking Based TCDS 
International Journal of Computer Science \& Information Technology (IJCSIT) Vol 7, No 2, April 2015

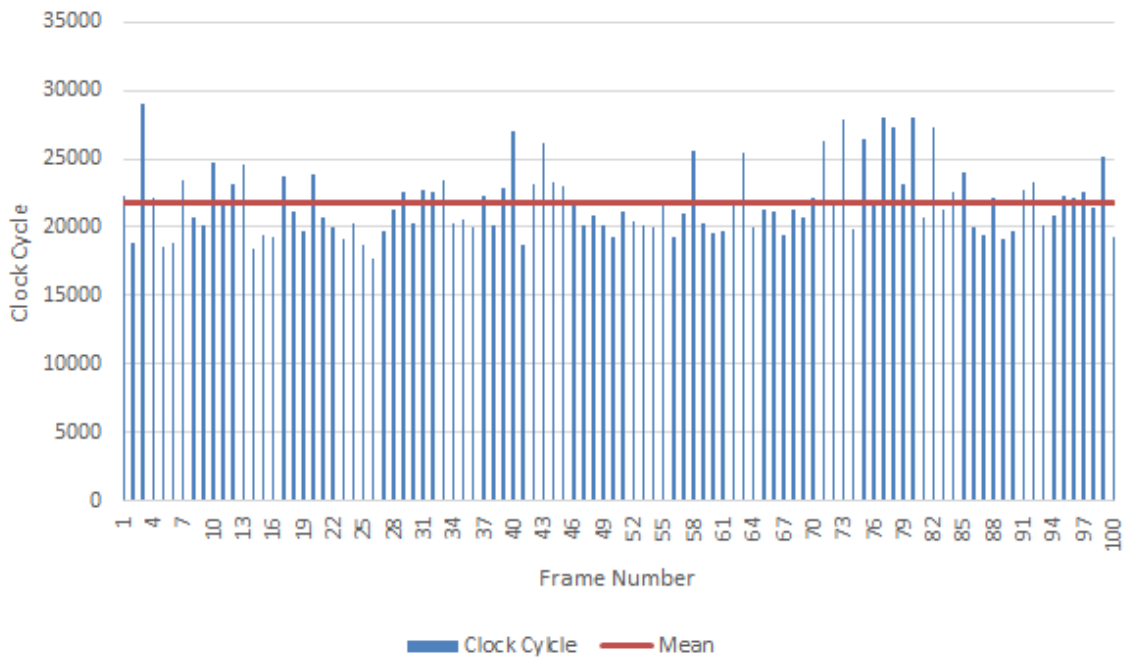

Figure 4.a - CPU Clock Cycle Performance of Histogram Based TCDS

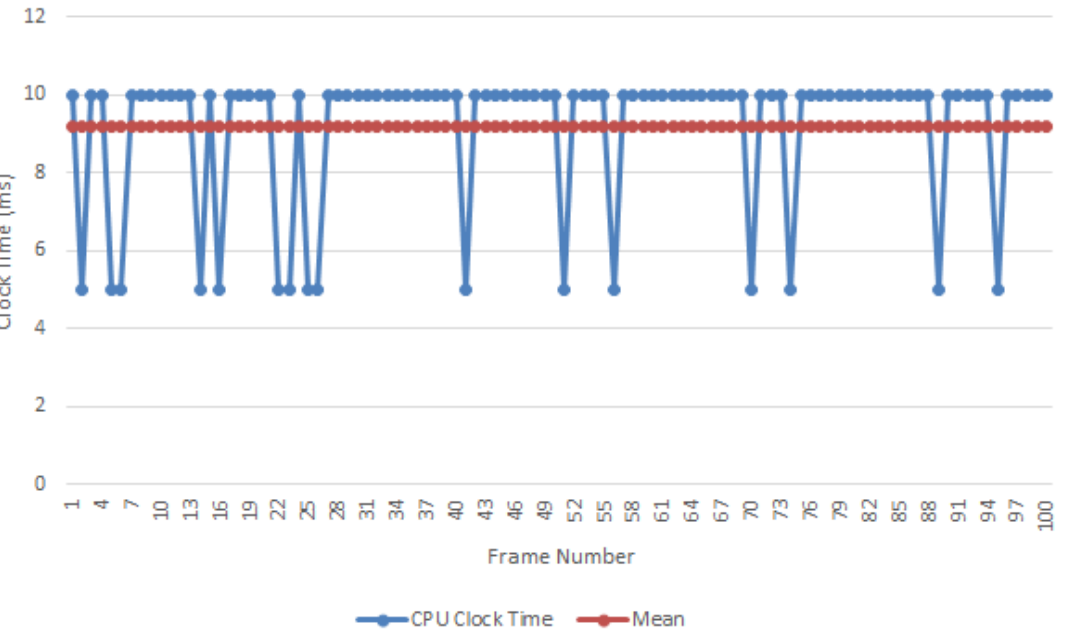

Figure 4.b - Execution Time Performance of Histogram Based TCDS

\begin{tabular}{|l|c|c|c|c|}
\hline \multirow{2}{*}{} & \multicolumn{2}{|c|}{ Feature Tracking } & \multicolumn{2}{c|}{ Histogram } \\
\cline { 2 - 5 } & Clock Cycle & Execution Time & Clock Cycle & Execution Time \\
\hline Minimum & 242318 & $95 \mathrm{~ms}$ & 17782 & $5 \mathrm{~ms}$ \\
\hline Maximum & 293180 & $115 \mathrm{~ms}$ & 28976 & $10 \mathrm{~ms}$ \\
\hline Average & 264119,4 & $103 \mathrm{~ms}$ & 21812,88 & $9.2 \mathrm{~ms}$ \\
\hline
\end{tabular}

Table 2 - Performance Comparison between Feature Tracking and Histogram based TCDS

Although developed both solutions support real-time execution, Figure 3 and Figure 4 and Table 2 indicate that the histogram based TCDS is more efficient than feature tracking TCDS while comparing these algorithms in real-time. 
The algorithms have been tested under different weather conditions such as cloudy, sunny and rainy to provide various illuminations at both daytime and night. While performing the feature tracking based solution both daytime and night, the success rate depends on the feature extracting success rate. Also, the success rate of histogram based solution depends on the illumination on the camera display. In our experiment, we have tested the both solutions under different illuminations at daytime (cloudy, sunny and rainy) and night (clear and rainy). Feature based solution provides accurate results under mentioned illumination conditions at both daytime and night. On the other hand, histogram based solution gives accurate results under mentioned illumination conditions at daytime. While performing the histogram based solution at rainy night, results can be affected from headlight of cars and light reflection causing from wet roads.

\section{CONCLUSION}

In this paper, feature tracking based and histogram based Traffic Congestion Detection System (TCDS) have been developed and implemented as a part of Traffic Surveillance System. The developed systems are designed to process in a real time manner. After that, performance of both systems are compared. In this context, the developed system have been tested under different illumination conditions at daytime and night. The obtained results show us, both solutions are suitable to detect the congestion at daytime. However, feature tracking TCDS is more accurate than histogram TCDS at the rainy night because of the light reflection. Also, CPU cycle and CPU time performance have been measured in order to point out the availability of real time execution. The obtained results show us, despite the two solutions support real-time execution, histogram based solution is more applicable to execute in real time. In this context we will design a system to combine this solutions to cover all conditions as a future work.

\section{REFERENCES}

[1] V. Jain, A. Sharma, and L. Subramanian, (2012) "Road traffic congestion in the developing world", Proceedings of the 2nd ACM Symposium on Computing for Development - ACM DEV '12, p. 1

[2] K. Peiris and D. Sonnadara, (2011) "Extracting Traffic Parameters at Intersections through Computer Vision", Proceedings of the Technical Sessions, vol. 27, pp. 68-75

[3] K. Robert, (2009) "Video-based traffic monitoring at day and night: Vehicle features detection and tracking", in IEEE Conference on Intelligent Transportation Systems, Proceedings, ITSC, 2009, pp. 285-290.

[4] B. Coifman, D. Beymer, P. McLauchlan, and J. Malik, (1998) "A real-time computer vision system for vehicle tracking and traffic surveillance", Transportation Research Part C: Emerging Technologies, vol. 6, no. 4, pp. 271-288

[5] E. Rublee, V. Rabaud, K. Konolige, and G. Bradski, (2011) "ORB: An efficient alternative to SIFT or SURF", Proceedings of the IEEE International Conference on Computer Vision, pp. 2564-2571.

[6] E. Rosten and T. Drummond, (2006) "Machine learning for high-speed corner detection", Lecture Notes in Computer Science (including subseries Lecture Notes in Artificial Intelligence and Lecture Notes in Bioinformatics), vol. 3951 LNCS, pp. 430-443.

[7] M. Calonder, V. Lepetit, C. Strecha, and P. Fua, (2010) "BRIEF: Binary robust independent elementary features ", Lecture Notes in Computer Science (including subseries Lecture Notes in Artificial Intelligence and Lecture Notes in Bioinformatics), vol. 6314 LNCS, no. PART 4, 2010, pp. 778-792.

[8] K. Peiris and D. Sonnadara, (2011) "Extracting Traffic Parameters at Intersections through Computer Vision", Proceedings of the Technical Sessions, vol. 27, pp. 68-75 


\section{Authors}

Ozgur Altun received the B.Sc. degree in Computer Engineering from Kocaeli University, Turkey, in 2010. He is received the M.Sc. degree in Satellite Communication and Remote Sensing at Istanbul Technical University in 2013. He is currently pursuing the $\mathrm{PhD}$ degree in Computer Engineering at Hacettepe University. He worked as a research assistant at Yeditepe University between 2010 and 2013. He is currently working in Proline Bilisim Sistemleri as a Research and Development Engineer. His research interests include computer vision, image processing, satellite image processing, wireless sensor networks, activity monitoring,

Kenan Aksoy received the B.Sc. degree in Computer Engineering from Başkent University, Turkey, in 2001. He worked as a software at Ekin Technology between 2005 and 2012. He is currently working in Proline Bilisim Sistemleri as a Research and Development Engineer. His research interests include computer vision, image processing, video protocols and format.
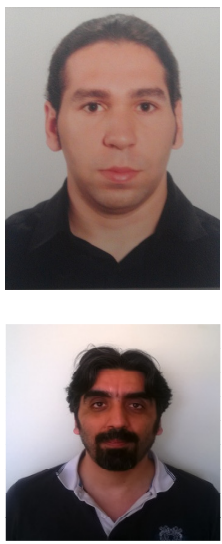